\title{
Erratum zu: Mathematische Begabung in den Sekundarstufen erkennen und angemessen aufgreifen
}

Benjamin Rott und Maike Schindler

Erratum zu:

Kapitel 20 in: J. Leuders et al. (Hrsg.), Mit Heterogenität im

Mathematikunterricht umgehen lernen https://doi.org/10.1007/978-3-658-16903-9_20

Während der Produktionsphase des Titels ist versehentlich die Aktualisierung einer Autorenadresse auf Seite 235 nicht vorgenommen worden.

Die korrekte Adresse lautet:

B. Rott

Mathematisch-Naturwissenschaftliche Fakultät, Institut für Mathematikdidaktik, Universität zu Köln

Köln, Deutschland

Die aktualisierte Version des Kapitels kann hier abgerufen werden:

https://doi.org/10.1007/978-3-658-16903-9_20

B. Rott $(\bowtie)$

Mathematisch-Naturwissenschaftliche Fakultät, Institut für Mathematikdidaktik, Universität zu Köln

Köln, Deutschland

M. Schindler

Humanwissenschaftliche Fakultät, Department Heilpädagogik und Rehabilitation, Universität zu Köln

Köln, Deutschland 